\title{
Correlation of the Cd-to-Te ratio on CdTe surfaces with the surface structure
}

\author{
Y. S. Wu, ${ }^{*}$ C. R. Becker, A. Waag, M. M. Kraus, R. N. Bicknell-Tassius, and G. Landwehr \\ Physikalisches Institut der Universität Würzburg, D-8700 Würzburg, Federal Republic of Germany
}

(Received 20 November 1990)

\begin{abstract}
We report here that reconstruction on (100), (111) $A$, and (111) $B$ CdTe surfaces is either $c(2 \times 2)$, $(2 \times 2)$, and $(1 \times 1)$ or $(2 \times 1),(1 \times 1)$, and $(1 \times 1)$ when they are $\mathrm{Cd}$ or Te stabilized, respectively. There is a mixed region between $\mathrm{Cd}$ and $\mathrm{Te}$ stabilization in which the reflected high-energy electron-diffraction (RHEED) patterns contain characteristics of both $\mathrm{Cd}$ - and Te-stabilized surfaces. We have also found that the Cd-to-Te ratio of the $\mathrm{x}$-ray photoelectron intensities of their $3 d_{3 / 2}$ core levels is about $20 \%$ larger for a Cd-stabilized (111) A, (111)B, or (100) CdTe surface than for a Te-stabilized one. According to a simple model calculation, which was normalized by means of the photoelectron intensity ratio of a Cd-stabilized (111) $A$ and a Te-stabilized (111)B CdTe surface, the experimental data for CdTe surfaces can be explained by a linear dependence of the photoelectron-intensity ratio on the fraction of $\mathrm{Cd}$ in the uppermost monatomic layer. This surface composition can be correlated with the surface structure, i.e., the corresponding RHEED patterns. This correlation can in turn be employed to determine $\mathrm{Te}$ and $\mathrm{Cd}$ evaporation rates. The Te reevaporation rate is increasingly slower for the Te-stabilized (111) $A,(111) B$, and (100) surfaces, while the opposite is true for Cd from Cd-stabilized (111) $A$ and (111) $B$ surfaces. In addition, Te is much more easily evaporated from all the investigated surfaces than is $\mathrm{Cd}$, if the substrate is kept at normal molecular-beam-epitaxy growth temperatures ranging from $200^{\circ} \mathrm{C}$ to $300^{\circ} \mathrm{C}$.
\end{abstract}

\section{INTRODUCTION}

The II-VI semiconductor alloys $\mathrm{Hg}_{1-x} \mathrm{Cd}_{x} \mathrm{Te}$, $\mathrm{Hg}_{1-x} \mathrm{Mn}_{x} \mathrm{Te}$, and $\mathrm{Hg}_{1-x} \mathrm{Zn}_{x} \mathrm{Te}$ are of great interest because of their use in infrared detectors. ${ }^{1-6} \mathrm{Cd}_{1-x} \mathrm{Mn}_{x} \mathrm{Te}$ and $\mathrm{Cd}_{1-x} \mathrm{Zn}_{x} \mathrm{Te}$ are also interesting since the former is a widely studied semimagnetic semiconductor and the latter is an extensively used, lattice-matched substrate for $\mathrm{Hg}$-Cd-Te alloys as well as a wide-gap semiconductor with a band gap that ranges from 1.5 to $2.3 \mathrm{eV}$ and thus has a potential as a tunable source, detector, or solar cell in the visible region. ${ }^{7-10}$ The basic substrate materials for molecular-beam-epitaxy (MBE) growth of such alloys are $\mathrm{CdTe}, \mathrm{Cd}_{1-x} \mathrm{Zn}_{x} \mathrm{Te}$, and to a lesser extent GaAs, whose crystalline quality is normally better than that of CdTe and $\mathrm{Cd}_{1-x} \mathrm{Zn}_{x}$ Te. However, a CdTe buffer is usually grown on the substrate, which must be thicker in the case of GaAs due to the large lattice mismatch. Therefore the MBE growth of high-quality CdTe is of the utmost importance. Consequently many workers ${ }^{2-16}$ have been involved in an attempt to improve the quality of $\mathrm{MBE}$-grown $\mathrm{CdTe}$ - and $\mathrm{Hg}$-based films.

Greater reproducibility in crystalline quality of MBEgrown GaAs has been achieved by correlating the bulk properties of MBE-grown GaAs to the surface stoichiometry. ${ }^{11}$ The structure of a real surface is always different from the structure of the truncated bulk material due to a rearrangement of the surface atoms. Thus the first layer has a different periodicity, which is strongly correlated to the bulk symmetry and which depends on the growth conditions.
For these reasons we have investigated the influence of various growth conditions on the CdTe surface. The method most widely employed to study the surface during $\mathrm{MBE}$ growth is reflection high-energy electron diffraction (RHEED). RHEED patterns can be used to observe surface reconstruction as a function of growth conditions such as source fluxes, substrate temperature, etc. $^{2,11-15}$ However, high-energy electrons (HEE) have been shown to influence the surface appreciably ${ }^{14}$ and thus one must be sure that the HEE are not changing what one is studying. To our knowledge, no publication has correlated RHEED patterns to the $\mathrm{Cd}$ and $\mathrm{Te}$ contents of the uppermost atomic layer or layers. Recently Benson et al. proposed that the half-order reconstruction (HOR) in the [011] azimuth is related to a Te-stabilized surface and HOR in the [001] and [010] azimuths is related to a Cd-stabilized surface. ${ }^{12}$ In principle, the concentrations of the surface layer or layers can be determined with the help of $\mathrm{x}$-ray photoelectron spectroscopy (XPS). However, there are some experimental difficulties such as uncertainty in the atomic sensitivity factors (ASF). ${ }^{16-20}$

In this investigation we determined the ratio of the photoelectron intensity of the $3 d_{3 / 2}$ core levels of $\mathrm{Cd}$ and $\mathrm{Te}$, corresponding to the RHEED pattern of a (100) $\mathrm{CdTe}$ surface ranging from a Cd-stabilized to a Testabilized surface. We propose a simple model that is normalized using the results of measurements on the (111) $A$ and (111) $B$ CdTe surfaces and that allows us to calculate a $\mathrm{Cd}$ fraction in the uppermost monatomic layer ( $\mathrm{Cd}$ surface coverage) and thus to correlate it to a RHEED pattern. XPS measurements have been carried out on (100) and (110) surfaces grown under different conditions. 


\section{EXPERIMENTAL DETAILS}

The experiments were performed using a four-chamber RIBER 2300 MBE system; two growth chambers for wide-band and narrow-band II-VI semiconductors, one XPS chamber, and one metallization chamber. These chambers are connected with each other by means of a transfer system. Normally the vacuum is better than $6 \times 10^{-10}$ and $2 \times 10^{-9}$ Torr in the narrow-band growth chamber and transfer system, respectively. The acceleration voltage of the RHEED electron gun was approximately $9 \mathrm{kV}$, the incident-electron-beam current was between 20 and $80 \mu \mathrm{A}$, and the beam was focused as small as possible. XPS experiments were performed with a RIBER MAC2 electron spectrometer using a $\mathrm{Mg} K \alpha \mathrm{x}$ ray source $(h v=1235.6 \mathrm{eV})$ with an acceleration voltage of $10 \mathrm{kV}$, a current of $10 \mathrm{~mA}$, and without a monochromator.

The substrates employed were mostly $\mathrm{Cd}_{1-x} \mathrm{Zn}_{x} \mathrm{Te}$ $(4 \% \mathrm{Zn})$ and some CdTe with (100), (110), (111) $A$, and (111) $B$ orientations. They were chemomechanically polished for several minutes, degreased using standard solvents, etched in a weak solution of bromine in methanol, and rinsed in methanol. Immediately prior to loading the substrates into the MBE system, they were rinsed in deionized water, briefly dipped in hydrochloric acid, and then rinsed in deionized water to remove any remaining carbon and all of the original oxide from the surface. The substrates were preheated at $100^{\circ} \mathrm{C}$ for $15 \mathrm{~min}$ and then the temperature was slowly raised to $340^{\circ} \mathrm{C}-350^{\circ} \mathrm{C}$, until the RHEED pattern indicated an absence of oxygen.

In order to obtain a smooth CdTe surface as determined by the RHEED pattern, i.e., long, uniform streaks, and thus a consistent starting point, we first grew a CdTe buffer at $300^{\circ} \mathrm{C}-350^{\circ} \mathrm{C}$ for $2 \mathrm{~h}$, which was about $1 \mu \mathrm{m}$ thick. A CdTe flux of $3.5 \times 10^{-7}$ Torr was used throughout this investigation for the growth of CdTe. The substrate temperature was lowered to $230^{\circ} \mathrm{C}$ and CdTe was allowed to grow for $1.5 \mathrm{~h}$, with an additional thickness of about $1 \mu \mathrm{m}$. In order to obtain a smooth CdTe surface on the (111) $A$ surface, a growth temperature of at least $320^{\circ} \mathrm{C}$ was required. Before a substrate was used again it was heated to $340^{\circ} \mathrm{C}$ for $10 \mathrm{~min}$ and a smooth surface was reestablished by growing a $\mathrm{CdTe}$ buffer for about $30 \mathrm{~min}$ at $230^{\circ} \mathrm{C}$, again with the exception of the (111) $\mathrm{A}$ surface, which required $340^{\circ} \mathrm{C}$. These are the normal starting materials for the experiments described below in the results from Secs. III A-III E, unless otherwise stated.

The substrate was then either kept in a Cd or Te environment in order to produce a Cd- or Te-stabilized surface, or heated to a higher temperature in order to obtain a mixed surface. The $C d$ and Te environments consisted of $\mathrm{Cd}$ and Te fluxes of $2 \times 10^{-7}$ and $3 \times 10^{-7}$ Torr, respectively. The only exceptions were a $\mathrm{Cd}$ flux of $1 \times 10^{-6}$ Torr for the (110) surface and a $\mathrm{Te}$ flux of $6 \times 10^{-7}$ Torr for the (111) $A$ surface. The photoelectron-intensity ratio $(Q)$ of $\mathrm{Cd}$ and $\mathrm{Te} 3 d_{3 / 2}$ core levels was measured at room temperature using the area of the corresponding peaks with an experimental error of about
$1 \%$. The $3 d_{3 / 2}$ core levels were employed rather than the $3 d_{5 / 2}$ core levels in order to avoid the $K \alpha$ satellites of $\mathrm{Mg}$.

\section{RESULTS}

Throughout this paper we consistently use the convention of referring to the direction of the incident electrons when referring to reconstruction in a particular azimuth. Normally, after preheating the (100) CdTe substrate,

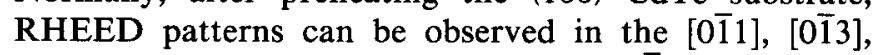

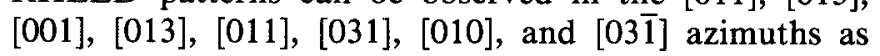
shown in Fig. 1 and in the opposite directions. No distinct HOR could be found on such a surface. However, during growth, HOR was observed in some of these azimuths. For normal growth conditions a strong HOR in the (011) azimuth and weak but clear HOR in the [03 $\overline{1}]$ azimuth were observed. Reconstruction was present in the [001], [013], [031], and [010] azimuths on a Cdstabilized surface, and in the [0 $\overline{1} 1],[0 \overline{1} 3],[011]$, and [03 $\overline{1}]$ azimuths on a Te-stabilized surface. Half-order reconstruction in the [03 $\overline{1}$ ] azimuth is accompanied by two weaker lines indicating the possibility of a fourfold reconstruction. RHEED observations for (111) CdTe are more complicated and are described below.

\section{A. (100) Te-stabilized surface}

In order to get a Te-stabilized CdTe surface we opened the Te shutter immediately after CdTe growth and kept the starting material in a Te environment for about $2 \mathrm{~min}$ until the sample temperature was less than $210^{\circ} \mathrm{C}$. Whereas HOR was very strong in the [011] azimuth, it was weaker but distinct in the [01ㅣ azimuth. If the reconstruction were pure $(2 \times 1)$ as reported in the literature, ${ }^{6,12}$ the HOR in the [011] azimuth should be absent instead of weaker. We therefore suggest that either the surface is not completely Te stabilized or the reconstruction is an approximation of $(2 \times 1)$ but will be hereafter designated as $(2 \times 1)$. The strength of HOR in the [03 $\overline{1}]$

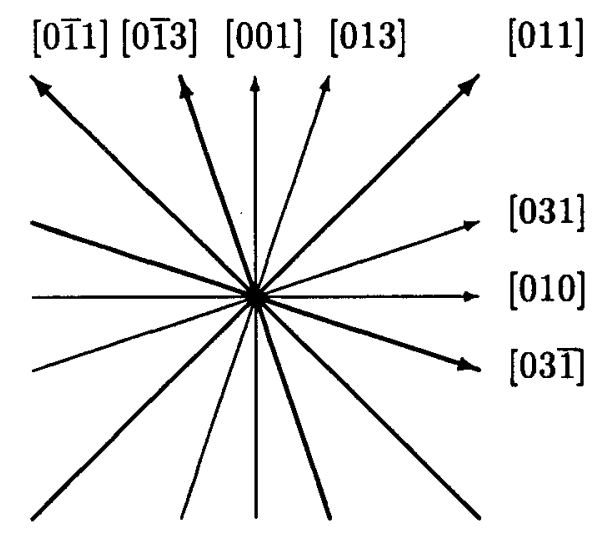

FIG. 1. The azimuths in which reconstruction can be observed in reflection high-energy electron-diffraction patterns of the (100) CdTe surface. The azimuths for a Te and Cd environment are indicated by thick and thin lines, respectively. 
azimuth was the same as that of the integral order streaks. It completely disappeared when in a Cd environment and therefore equal HOR and integral order intensities were considered to signify a Te-stabilized surface. At room temperature we found no change in the RHEED pattern. This is consistent with the fact that the desorption time of Te atoms from the surface at $210^{\circ} \mathrm{C}$ is at least several hours, according to the measurements of Benson et al. ${ }^{12}$ and Wu et al. ${ }^{13,14}$ From XPS measurements the photoelectron-intensity ratio $Q$ was determined to be 0.430 and is listed in Table I.

\section{B. (100) Cd-stabilized surface}

Similarly, a Cd-stabilized surface was established by exposing the starting material to a $\mathrm{Cd}$ environment until the substrate temperature was below $180^{\circ} \mathrm{C}$. After closing the $\mathrm{Cd}$ shutter and the main shutter, the substrate was held at $180^{\circ} \mathrm{C}$ for several minutes, and then cooled to room temperature where the intensity of the HOR between the zero- and first-order Laue zones was the same as that of the HOR between the first- and second-order Laue zones in the [010] azimuth. We used this equality of HOR intensities to signify a Cd-stabilized surface. No HOR was observed in the [03i] azimuth. At the present we ignore HOR in the [011] azimuth because it was very weak and could not be completely removed. We attempted to produce HOR in the [010] azimuth without HOR in the [011] azimuth by keeping $\mathrm{CdTe}$ at $150^{\circ} \mathrm{C}$ in a larger Cd flux. After the RHEED patterns disappeared, which occurred within several seconds, we slowly increased the substrate temperature until HOR appeared in the [010] and [011] azimuths simultaneously.

We have also preheated several CdTe substrates at $350^{\circ} \mathrm{C}-400^{\circ} \mathrm{C}$ for $15 \mathrm{~min}$ and then cooled them to $150^{\circ} \mathrm{C}$ in a $\mathrm{Cd}$ environment. Obviously such substrates should have a very rough surface. Their RHEED patterns were spotty and no reconstruction could be found. But their intensity ratio $Q$ was about 0.50 , which is almost the same as that for a smooth Cd-stabilized surface, 0.505 .

\section{C. (100) Cd-Te mixed surface}

Surfaces intermediate to a Te- and a Cd-stabilized surface, as judged by the RHEED patterns and their intensity ratios obtained from XPS measurements, were ob- tained by keeping the starting material at a particular temperature without either a $\mathrm{Te}$ or $\mathrm{Cd}$ flux. The three surfaces that result at temperatures of $340^{\circ} \mathrm{C}, 280^{\circ} \mathrm{C}$, and $230^{\circ} \mathrm{C}$ are considered below and the experimental results are also listed in Table $\mathrm{I}$.

The first surface was acquired by growing $\mathrm{CdTe}$ at $340^{\circ} \mathrm{C}$ for $20 \mathrm{~min}$ and then allowing the sample to cool to room temperature after stopping the growth. HOR between the first- and second-order Laue zones in the [010] azimuth was still strong. HOR in the $[03 \overline{1}]$ azimuth was present but very weak. Using a smaller $\mathrm{Cd}$ flux at lower temperatures can also produce such a surface structure.

In the second case CdTe was grown for several minutes with the substrate at $280^{\circ} \mathrm{C}$. HOR was weaker between the first- and second-order Laue zones in the [010] azimuth but stronger and clearer in the [03ī] azimuth than in the first case.

The third surface was that of our normal starting material, i.e., CdTe grown at $230^{\circ} \mathrm{C}$ for $30 \mathrm{~min}$ and cooled to room temperature after growth. HOR in the [010] azimuth completely disappeared, was very strong in the [011] azimuth, and was very clear in the [03ī] azimuth. The surface was very smooth as indicated by the uniform streaks in the RHEED pattern.

It is clear from Table I that as the intensity ratio $Q$ decreases, HOR in the [011] azimuth becomes stronger while HOR in the [031] azimuth increases in intensity until it is the same as that of the integral streaks. In contrast, HOR in the [010] azimuth decreases in intensity until it disappears completely. HOR in the [011] azimuth is always present if the surface is smooth enough. There-

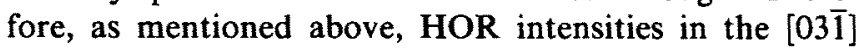
and [010] azimuths are an indication of $\mathrm{Te}-$ and $\mathrm{Cd}-$ stabilized surfaces, respectively.

\section{D. (110) CdTe surface}

The (110) face is a nonpolar face, as shown in Fig 2. This face is ideally terminated by $\mathrm{Cd}$ and $\mathrm{Te}$ atoms, each with a single dangling bond at the surface where nucleation and growth occurs. We grew CdTe on the (110) CdTe substrate at a temperature ranging from $150^{\circ} \mathrm{C}$ to $350^{\circ} \mathrm{C}$ and no reconstruction other than $(1 \times 1)$ was found. The $(1 \times 4)$ reconstruction, as found by Arias, Shin, and Gertner, on (110) $\mathrm{Hg}_{1-x} \mathrm{Cd}_{x} \mathrm{Te}$ substrates, ${ }^{21}$

TABLE I. The photoelectron-intensity (peak area) ratio $Q$ of the $3 d_{3 / 2}$ core level of $\mathrm{Cd}$ and Te atoms in (100) CdTe, as well as the directions in which reconstruction was observed in the reflection high-energy electron-diffraction (RHEED) patterns for different experimental conditions. An asterisk denotes weak but distinct reconstruction.

\begin{tabular}{|c|c|c|c|c|c|}
\hline $\begin{array}{c}Q(\mathrm{Cd}: \mathrm{Te}) \\
\text { Conditions }\end{array}$ & $\begin{array}{c}0.505 \\
\text { Cd stabilized } \\
\end{array}$ & $\begin{array}{c}0.480 \\
T_{s}=340^{\circ} \mathrm{C} \\
\end{array}$ & $\begin{array}{c}0.460 \\
T_{s}=280^{\circ} \mathrm{C} \\
\end{array}$ & $\begin{array}{c}0.440 \\
T_{s}=230^{\circ} \mathrm{C}\end{array}$ & $\begin{array}{c}0.430 \\
\text { Te stabilized } \\
\end{array}$ \\
\hline $\begin{array}{l}\text { The azimuths in } \\
\text { which reconstruction } \\
\text { was observed } \\
\text { in the RHEED } \\
\text { patterns }\end{array}$ & $\begin{array}{l}{[010]} \\
{[001]} \\
{[013]} \\
{[031]} \\
{[011]^{*}}\end{array}$ & $\begin{array}{l}{[010]} \\
{[001]} \\
{[013]} \\
{[031]} \\
{[011]^{*}} \\
{[031]^{*}} \\
{[013]^{*}}\end{array}$ & $\begin{array}{l}{[010]} \\
{[001]} \\
{[013]} \\
{[031]} \\
{[011]} \\
{[03 \overline{1}]} \\
{[0 \overline{1} 3]}\end{array}$ & $\begin{array}{l}{[011]} \\
{[03 \overline{1}]} \\
{[0 \overline{1} 3]} \\
{[0 \overline{1} 1]^{*}}\end{array}$ & $\begin{array}{l}{[011]} \\
{[03 \overline{1}]} \\
{[0 \overline{1} 3]} \\
{[0 \overline{1} 1]^{*}}\end{array}$ \\
\hline
\end{tabular}




\section{(110) face}

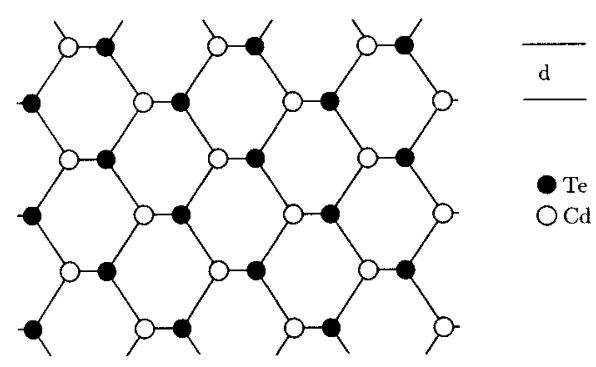

FIG. 2. Crystal structure of (110) CdTe: O, Cd; $\bullet, T e$.

could not be found by either keeping the substrate in a $\mathrm{Cd}$ environment at $80^{\circ} \mathrm{C}$ or in a $\mathrm{Te}$ environment at $160^{\circ} \mathrm{C}$. An intensity ratio of $0.466 \pm 0.005$ was obtained using various conditions, such as subjecting the substrate to a $\mathrm{Cd}$ or $\mathrm{Te}$ environment for several minutes, as long as the RHEED pattern indicated a smooth surface.

\section{E. (111) $A$ and (111) $B$ CdTe surfaces}

The (111) face in the zinc-blende structure is a polar face. This polarity leads to two types of faces, as shown in Fig. 3. The first is the (111) $A$ face. It is terminated by either a triply bonded $\mathrm{Cd}$ atom or a singly bonded $\mathrm{Te}$ atom and is called the $\mathrm{Cd}$ face since the stable configuration is terminated by $\mathrm{Cd}$ atoms. We grew $\mathrm{CdTe}$ at temperatures ranging from $340^{\circ} \mathrm{C}$ to $200^{\circ} \mathrm{C}$ but found clear $(2 \times 2)$ reconstruction, which is typical of a $\mathrm{CdTe}$ surface in a Cd environment, only during growth at temperatures between $320^{\circ} \mathrm{C}$ and $340^{\circ} \mathrm{C}$. After growth reconstruction became stronger and after $10 \mathrm{~h}$ at $230^{\circ} \mathrm{C}$ in the growth chamber, the HOR strength was the same as that of the integral streaks and the RHEED pattern was smoother. The intensity ratio $Q$ was 0.509 and the RHEED patterns had not changed after the XPS measurement.

However, if kept in a Te environment until the temperature was $120^{\circ} \mathrm{C}$ or less, reconstruction was $(1 \times 1)$ and the measured $Q$ was 0.402 . High-energy electrons reestablished $(2 \times 2)$ reconstruction in a Te environment in a very short time, decreasing to nearly zero at higher substrate temperatures. This behavior was observed at temperatures as low as $70^{\circ} \mathrm{C}$. In the absence of HEE and a

(111)B face

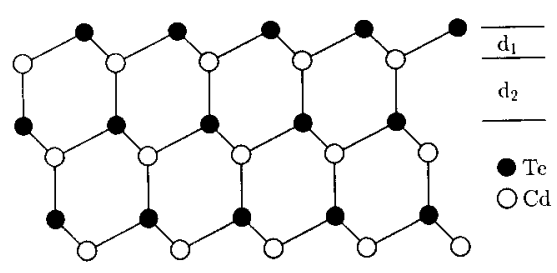

(111)A face

FIG. 3. Crystal structure of (111) CdTe: O, Cd; $\bullet$ Te.
Te environment, $(1 \times 1)$ reconstruction persisted for $10-20 \mathrm{~s}$ at $150^{\circ} \mathrm{C}$ before becoming $(2 \times 2)$. HEE irradiation was avoided, or more precisely limited to a period of $2-3 \mathrm{~s}$, by means of a switch that allowed the $x-y$ deviation voltages of the electron gun to be changed rapidly.

The other possible face, the (111) $B$ face, is terminated by $\mathrm{Te}$ atoms. ${ }^{15}$ During CdTe growth we observed $(2 \sqrt{3} \times 2 \sqrt{3}) R 30^{\circ}$ reconstruction, which became stronger after stopping growth, as reported by Sivananthan et al., ${ }^{15}$ Hsu et al., ${ }^{16}$ and Benson and Summers. ${ }^{22}$ However, in a Te environment the $(2 \sqrt{3} \times 2 \sqrt{3}) R 30^{\circ}$ RHEED pattern immediately disappeared and was transformed into a very clear and smooth $(1 \times 1)$ reconstruction if the sample was not being irradiated by HEE. Even though $(1 \times 1)$ reconstruction in a Te environment could be changed to a $(2 \sqrt{3} \times 2 \sqrt{3}) R 30^{\circ}$ reconstruction in about $10 \mathrm{~s}$ after the Te shutter was closed at $230^{\circ} \mathrm{C}$, at least 10 min was required in the $\mathrm{Cd}$ case at $230^{\circ} \mathrm{C}$. This is contrary to what one would expect because the (111)B is normally terminated with $\mathrm{Te}$ atoms.

After growing at $230^{\circ} \mathrm{C}$ for half an hour, the intensity ratio $Q$ ranged from 0.480 to 0.495 , increasing with the time that the film was held at a temperature of more than $200^{\circ} \mathrm{C}$. In contrast, if the substrate was subjected to a $\mathrm{Te}$ environment until being cooled to $150^{\circ} \mathrm{C}$ or less, which maintained the $(1 \times 1)$ reconstruction, a $Q$ of 0.433 was measured. Even though growth in a $\mathrm{Cd}$ environment is more difficult, $(1 \times 1)$ reconstruction was also observed on the (111) $B$ face in a Cd environment, as observed by Benson and Summers. ${ }^{22}$ Its intensity ratio $Q$ was 0.505 . (1X1) reconstruction in a $\mathrm{Te}$ environment on both (111) $A$ and (111) $B$ was easily removed by HEE in a matter of seconds. It is obvious that HEE employed in RHEED observations have a large effect on these surfaces. This may be the reason why $(1 \times 1)$ reconstruction has not been reported before.

\section{MODEL FOR THE CALCULATION OF THE Cd FRACTION ON CdTe SURFACES}

In this model we assume that a Cd-stabilized (111) $A$ surface and a Te-stabilized (111) $B$ surface are completely covered with one monatomic layer of $\mathrm{Cd}$ or $\mathrm{Te}$, respectively. This allows us to calculate a surface ASF ratio for the $\mathrm{Cd}$ and $\mathrm{Te} 3 d_{3 / 2}$ core levels. Using this ratio, the $\mathrm{Cd}$ fraction on the (100) and (110) surfaces can be determined. This calculation is described in detail below and the results will be compared with experiment.

In terms of XPS theory, the attenuation of the photoelectron flux through inelastic scattering can be described as follows. If $I_{0}(x)$ is the photoelectron flux, at a particular electron kinetic energy $E$, originating at a depth $x$ below the surface of a solid, the flux $I(x)$ emerging from the surface is given by

$$
I(x)=I_{0}(x) \exp \left[\frac{-x}{\lambda}\right),
$$

where $\lambda$ is the mean escape depth of an electron of energy $E$ within the material concerned.

Thus flux, which experimentally is the peak area of the 
$3 d_{3 / 2}$ core level of either $\mathrm{Cd}$ or $\mathrm{Te}$, is given for a (111) $\mathrm{A}$ surface by

$$
\begin{aligned}
I_{\mathrm{Cd}} & =N I_{\mathrm{Cd}}(0) \exp \left[\frac{-d_{\mathrm{Cd}}}{\lambda_{\mathrm{Cd}}}\right]_{n=0}^{n=\infty} \exp \left[\frac{-n\left(d_{1}+d_{2}\right)}{\lambda_{\mathrm{Cd}}}\right] \\
& =N I_{\mathrm{Cd}}(0)-\frac{\exp \left(\frac{-d_{\mathrm{Cd}}}{\lambda_{\mathrm{Cd}}}\right)}{1-\exp \left(\frac{-d_{1}-d_{2}}{\lambda_{\mathrm{Cd}}}\right)}, \\
I_{\mathrm{Te}} & =N I_{\mathrm{Te}}(0) \exp \left(\frac{-d_{\mathrm{Cd}}-d_{1}}{\lambda_{\mathrm{Te}}} \sum_{n=0}^{n=\infty} \exp \left(\frac{-n\left(d_{1}+d_{2}\right)}{\lambda_{\mathrm{Te}}}\right)\right. \\
& =N I_{\mathrm{Te}}(0) \frac{\exp \left(\frac{-d_{\mathrm{Cd}}-d_{1}}{\lambda_{\mathrm{Te}}}\right)}{1-\exp \left(\frac{-d_{1}-d_{2}}{\lambda_{\mathrm{Te}}}\right)},
\end{aligned}
$$

where $I_{\mathrm{Cd}}(0)$ and $I_{\mathrm{Te}}(0)$ are the photoelectron intensities produced by one $\mathrm{Cd}$ and one $\mathrm{Te}$ atom, respectively. $N$ is the number of atoms in one monatomic layer, $d_{1}$ and $d_{2}$ are the distances between neighboring layers and are equal to 0.935 and $2.806 \AA$, respectively, as shown in Fig. 3 , and $d_{\mathrm{Cd}}$ is the electron cloud thickness of the top layer, which we assume to be the covalent radius of $\mathrm{Cd}, 1.48 \AA$.

The photoelectron-intensity ratio for a Cd-stabilized (111) $A$ surface, $Q_{\mathrm{Cd}}[(111) A]$, is then

$$
\begin{aligned}
Q_{\mathrm{Cd}}(111) A= & \frac{I_{\mathrm{Cd}}}{I_{\mathrm{Te}}} \\
= & \frac{I_{\mathrm{Cd}}(0)}{I_{\mathrm{Te}}(0)}\left(\frac{1-\exp \left(\frac{\left(-d_{1}-d_{2}\right)}{\lambda_{\mathrm{Te}}}\right)}{1-\exp \left(\frac{\left(-d_{1}-d_{2}\right)}{\lambda_{\mathrm{Cd}}}\right)}\right) \\
& \times \exp \left(\frac{d_{\mathrm{Cd}}+d_{1}}{\lambda_{\mathrm{Te}}}-\frac{d_{\mathrm{Cd}}}{\lambda_{\mathrm{Cd}}}\right) .
\end{aligned}
$$

Similarly, for a Te-stabilized surface on the (111)B surface,

$$
\begin{aligned}
Q_{\mathrm{Te}}(111) B= & \frac{I_{\mathrm{Cd}}}{I_{\mathrm{Te}}} \\
= & \frac{I_{\mathrm{Cd}}(0)}{I_{\mathrm{Te}}(0)}\left(\frac{1-\exp \left(\frac{\left(-d_{1}-d_{2}\right)}{\lambda_{\mathrm{Te}}}\right)}{\left.1-\exp \left(\frac{\left(-d_{1}-d_{2}\right)}{\lambda_{\mathrm{Cd}}}\right)\right)}\right) \\
& \times \exp \left(\frac{d_{\mathrm{Te}}}{\lambda_{\mathrm{Te}}}-\frac{d_{\mathrm{Te}}+d_{1}}{\lambda_{\mathrm{Cd}}}\right)
\end{aligned}
$$

where $d_{\mathrm{Te}}$ has been taken to be the covalent radius of Te, $1.36 \AA$.

Equations (4) and (5) contain the factor $I_{\mathrm{Cd}}(0) / I_{\mathrm{Te}}(0)$, which is the ratio of the surface ASF for Cd and Te and which depends upon the instrument and the material. Therefore one would like to eliminate this factor. In order to do this we have divided Eq. (4) by Eq. (5), resulting in

$$
\begin{aligned}
R= & Q_{\mathrm{Cd}}(111) A / Q_{\mathrm{Te}}(111) B, \\
R= & \exp \left[\left(d_{\mathrm{Te}}-d_{\mathrm{Cd}}\right)\left(1 / \lambda_{\mathrm{Cd}}-1 / \lambda_{\mathrm{Te}}\right)\right] \\
& \times \exp \left[d_{1}\left(1 / \lambda_{\mathrm{Cd}}+1 / \lambda_{\mathrm{Te}}\right)\right] .
\end{aligned}
$$

If one uses the atomic radii or even half of the average distance between neighboring atomic layers for $d_{\mathrm{Cd}}$ and $d_{\mathrm{Te}}$, both $Q$ and $R$ are changed by less than $0.3 \%$. Values in the literature for the photoelectron escape depth for Cd $3 d_{3 / 2}$ core levels range from 15 to $18 \AA . .^{23,24}$ The corresponding value for $\mathrm{Te}$ was calculated using the relationship of $\lambda \sim E^{0.75}$. ${ }^{23}$ Using this range of values for the escape depths results in an uncertainty in $Q$ and $R$ of $\pm 1 \%$ and $\pm 3 \%$, respectively.

Assuming this model is correct for $\mathrm{Cd}$ - and Testabilized surfaces and normalizing it with $\mathrm{Cd}$ - and $\mathrm{Te}$ stabilized (111) $A$ and (111) $B$ surfaces, respectively, i.e., setting the experimental values for $Q_{\mathrm{Cd}}(111) A$ and $Q_{\mathrm{Te}}(111) B$ in Eqs. (4) and (5), the factor $I_{\mathrm{Cd}}(0) / I_{\mathrm{Te}}(0)$ was determined to be $0.395 \pm 0.009$. This factor can in turn be used to calculate $Q$ for other $\mathrm{Cd}$ - and Testabilized surfaces, e.g., $Q_{\mathrm{Cd}}(100), Q_{\mathrm{Te}}(100), Q_{\mathrm{Cd}}(111) B$, and $Q_{\mathrm{Te}}(111) A$, which are listed in Tables I and II along with their experimental values and their photoelectronintensity ratios.

Furthermore, both the calculated and measured values for $R$ corresponding to the $\mathrm{Cd}$ - and Te-stabilized surfaces on (100), (110), (111) $A$, and (111) $B$ faces are reproduced in Table III.

It has been established by low-energy electron diffraction (LEED), electron energy-loss spectroscopy (EELS), and theoretical calculations that the (110) surface of CdTe undergoes a $(1 \times 1)$ reconstruction in which the Te atoms at the surface move out by $0.18 \AA$ and the $\mathrm{Cd}$ atoms move in by $0.64 \AA . .^{25,26}$ Here we have ignored this

TABLE II. Experimental and theoretical values of the photoelectron-intensity (peak area) ratio $Q$ of the $3 d_{3 / 2}$ core level of $\mathrm{Cd}$ and Te atoms for Cd- and Te-stabilized (100), (111) $A$, (111)B, and (110) CdTe surfaces, e.g., $Q_{\mathrm{Te}}(111) A$ is the $\mathrm{Cd}$ to Te intensity ratio of the Te-stabilized (111) $A$ surface.

\begin{tabular}{llc}
\hline \hline & Experimental & Theoretical \\
\hline$Q_{\mathrm{Te}}(100)$ & $0.430 \pm 0.005$ & $0.424 \pm 0.004$ \\
$Q_{\mathrm{Cd}}(100)$ & $0.505 \pm 0.005$ & $0.527 \pm 0.005$ \\
$Q_{\mathrm{Te}}(11) A$ & $0.402 \pm 0.005$ & $0.393 \pm 0.006$ \\
$Q_{\mathrm{Cd}}(111) A$ & $0.509 \pm 0.005$ & $0.503 \pm 0.006$ \\
$Q_{\mathrm{Te}}(111) B$ & $0.433 \pm 0.005$ & $0.438 \pm 0.005$ \\
$Q_{\mathrm{Cd}}(11) B$ & $0.505 \pm 0.005$ & $0.573 \pm 0.010$ \\
$Q_{\mathrm{Te}}(110)$ & $0.466 \pm 0.005$ & $0.465 \pm 0.001$ \\
$Q_{\mathrm{Cd}}(110)$ & $0.466 \pm 0.005$ & $0.466 \pm 0.001$ \\
\hline
\end{tabular}


TABLE III. The quotient $R$ of the photoelectron-intensity ratio $Q$ of one Cd-stabilized surface to that of a Te-stabilized surface.

\begin{tabular}{lcc}
\hline \hline & Experimental & Theoretical \\
\hline$Q_{\mathrm{Cd}}(100) / Q_{\mathrm{Te}}(100)$ & $1.17 \pm 0.02$ & $1.24 \pm 0.03$ \\
$Q_{\mathrm{Cd}}(11) A / Q_{\mathrm{Te}}(111) B$ & $1.16 \pm 0.02$ & $1.15 \pm 0.03$ \\
$Q_{\mathrm{Cd}}(111) A / Q_{\mathrm{Te}}(111) A$ & $1.27 \pm 0.02$ & $1.28 \pm 0.03$ \\
$Q_{\mathrm{Cd}}(111) B / Q_{\mathrm{Te}}(111) B$ & $1.16 \pm 0.02$ & $1.31 \pm 0.03$ \\
$Q_{\mathrm{Te}}(110) / Q_{\mathrm{Cd}}(110)$ & $1.00 \pm 0.02$ & $1.00 \pm 0.01$ \\
\hline
\end{tabular}

effect since the photoelectron intensity should not depend upon the position of the atoms on the surface.

The experimental and theoretical values for both $Q$, the photoelectron-intensity ratio, and $R$, the ratio of $Q$ for a Cd-stabilized surface to $Q$ for a Te-stabilized surface fit well with the exception of $Q_{C d}(111) B$ and of course $Q_{\mathrm{Cd}}(111) B / Q_{\mathrm{Te}}(111) B$, which will be discussed below.

In Fig. 4 the photoelectron-intensity ratio $Q$ for the (100) surface is plotted versus the Cd surface coverage, i.e., the fraction of $\mathrm{Cd}$ in the uppermost monatomic layer. Here the theoretical values for a $\mathrm{Cd}$ - and Te-stabilized (100) surface are connected by a solid line and the theoretical uncertainties are represented by two dotted lines. The experimental values for $Q$ are represented by circles with error bars. A theoretical Cd surface coverage can be read directly from Fig. 4 for an experimental $Q$ value. Thus the $\mathrm{Cd}$ surface coverage is $5 \%$ and $78 \% \pm 6 \%$ for $\mathrm{Te}$ - and Cd-stabilized (100) CdTe surfaces, respectively.

Reconstruction in the RHEED patterns for the (100) surface with nearly equal amounts of $\mathrm{Cd}$ and $\mathrm{Te}$ occurs in all of the above-mentioned directions, which are listed in Table I. This situation is nearly the same as that observed during the growth of conducting CdTe films using

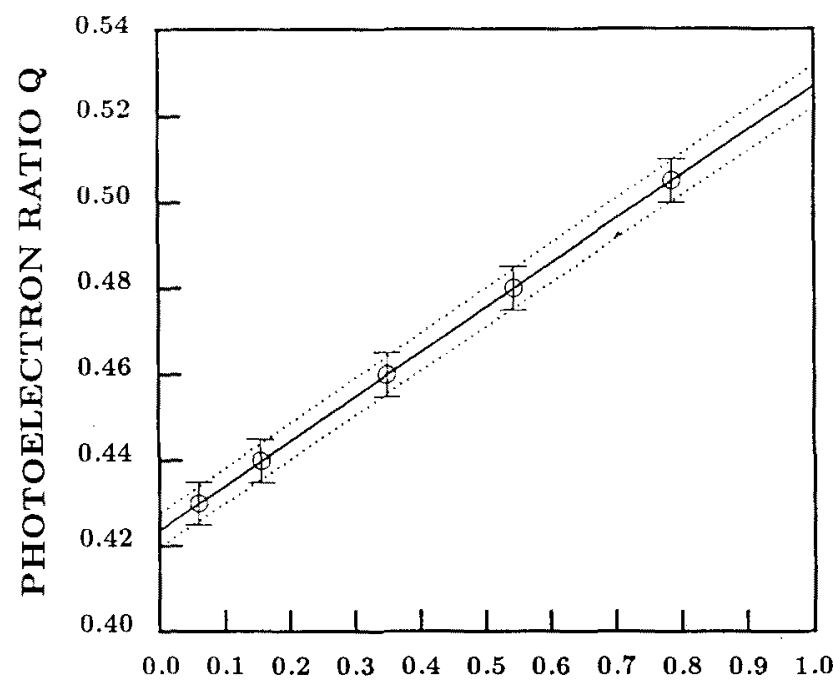

Cd SURFACE COVERAGE (ML)

FIG. 4. The theoretical Cd fraction on the $(100) \mathrm{CdTe}$ surface vs the photoelectron-intensity ratio as measured by $\mathrm{X}$-ray photoelectron spectroscopy. excess Cd flux. ${ }^{13}$ According to RHEED, the surface is smooth and displays characteristics of both a Cd- and Te-stabilized surface, i.e., HOR in the [011], [010], and [001] azimuths appear equally strong. This is possible if $\mathrm{Cd}$ - and Te-stabilized areas are connected by small steps.

\section{DISCUSSION}

The growth of high-quality $\mathrm{CdTe}$ or $\mathrm{Hg}_{1-x} \mathrm{Cd}_{x} \mathrm{Te}$ films requires a knowledge of the substrate surface, i.e., concentrations and structure, as determined by methods such as RHEED, XPS, etc. But it is difficult at best to measure the actual surface concentrations. If one uses XPS measurements, then one is confronted with large uncertainties in the atomic sensitivity factors due to differences in XPS instruments and standards.

$\mathrm{Lu}$, Feigelson, and Route studied CdTe (111) surfaces using angle-resolved XPS, Auger electron spectroscopy, and low-energy electron diffraction. ${ }^{19}$ They used ASF from the VG ESCALAB handbook to normalize their XPS data and found that $\mathrm{Cd}$ and $\mathrm{Te}$ compositions on the (111) $A$ and (111) $B$ surfaces were almost the same, $50.0 \% \pm 0.5 \%$. However, their samples were sputtered using a $1-\mathrm{kV}$ Ar ion beam for $10 \mathrm{~min}$ before being annealed at $300^{\circ} \mathrm{C}$ for $5 \mathrm{~min}$. Obviously these surfaces have been prepared much differently than ours and no comparison should be made.

Hsu et $a l .{ }^{16}$ used thick polycrystalline layers of CdTe, which they proposed to be stoichiometric in order to normalize their XPS measurements of (111)B CdTe. They did not describe the growth conditions under which these layers were grown. However, we have found that polycrystalline CdTe grown on (100) CdTe substrates at room temperature, $130^{\circ} \mathrm{C}$, and $160^{\circ} \mathrm{C}$ have a Te-rich surface, i.e., have a photoelectron-intensity ratio of $0.42 \pm 0.01$; see Fig. 4.

A previous investigation with a RIBER MAC2 electron spectrometer utilized pure $\mathrm{Cd}$ and $\mathrm{Te}$ on molybdenum in order to normalize the photoelectron intensity of the $3 d_{5 / 2}$ core level of $\mathrm{Cd}$ and $\mathrm{Te}$, and thus to obtain an ASF ratio of $0.83 .{ }^{18}$ Using this ASF ratio resulted in a Cd-to-Te ratio of 0.69 to 0.51 in a thin CdTe film with a thickness of 38 to $10 \AA$. Lu, Feigelson, and Route ${ }^{19}$ and Ekawa et al. ${ }^{20}$ used an ASF ratio of 0.73 and 0.83 , respectively, to normalize their XPS data for $3 d_{5 / 2}$ core levels of Cd and Te on a VG ESCALAB MK II and on a SSX-100, respectively.

In this investigation we have normalized the photoelectron-intensity ratios for the $3 d_{3 / 2}$ core levels by assuming that Cd-stabilized (111) $A$ and Te-stabilized (111) $B$ surfaces are covered by one monatomic layer of $\mathrm{Cd}$ and $\mathrm{Te}$, respectively. The experimental and theoretical values of the photoelectron-intensity ratio $Q$ are in good agreement. The only exception is the value of $Q$ for the Cd-stabilized (111)B surface, which can be explained if the singly bonded $\mathrm{Cd}$ atoms on the Cd-stabilized (111)B surface have undergone a rearrangement, which is not taken into account by our model. Another possibility is that the surface is not completely Cd stabilized, even with Cd fluxes up to $1 \times 10^{-6}$ Torr.

Subjecting the (111)B CdTe surface to a large Te flux, 
$4 \times 10^{-7}$ Torr, at $210^{\circ} \mathrm{C}$ resulted in $(1 \times 1)$ reconstruction. According to our XPS measurements the surface was $\mathrm{Te}$ stabilized with a photoelectron-intensity ratio $Q$ of 0.433. Benson and Summers observed $(2 \sqrt{3} \times 2 \sqrt{3}) R 30^{\circ}$ but not $(1 \times 1)$ reconstruction on the (111) $B$ CdTe surface, probably a result of the higher temperature of $300^{\circ} \mathrm{C}$, a much smaller $\mathrm{Te}$ flux and possibly the effect of HEE. ${ }^{22}$

Because of the good agreement between the experimental and theoretical values of the photoelectron-intensity ratio $Q$, the surface composition can be correlated with the surface structure, i.e., the corresponding RHEED patterns, as illustrated in Table I. This correlation can in turn be employed to determine $\mathrm{Te}$ and $\mathrm{Cd}$ evaporation rates as judged by the appropriate changes in the reconstruction.

In this manner we have found that $\mathrm{Te}$ reevaporates from a $(1 \times 1)$ Te-stabilized (111) $A$ surface very rapidly and indeed faster than from a (111) $B$ or (100) surface, i.e., the reevaporation times are $1 \mathrm{~s}$ or less, $10 \mathrm{~s}$ and $3 \mathrm{~h}$ at $230^{\circ} \mathrm{C}$, respectively. The reevaporation of $\mathrm{Cd}$ from the Cd-stabilized (111) $A$ face is much slower than from the Cd-stabilized (111)B, i.e., we see no change after $10 \mathrm{~h}$ for the former surface as compared to an evaporation time of less than $10 \mathrm{~min}$ for the latter surface. Sivananthan et al. have reasoned that since the (111) $A$ and (111) $B$ surfaces are terminated by triply bonded $\mathrm{Cd}$ and $\mathrm{Te}$, respectively, $\mathrm{Te}$ should act as a cap for $\mathrm{Cd}$ and $\mathrm{Hg}$ on the (111)B surfaces of $\mathrm{CdTe}$ and $\mathrm{Hg}_{1-x} \mathrm{Cd}_{x} \mathrm{Te}$, and thus that $\mathrm{Hg}$ and $\mathrm{Cd}$ should reevaporate more easily from the (111) $\mathrm{A}$ surface than from the (111) $B$ surface. ${ }^{15}$ However, we have observed the opposite behavior for $\mathrm{Cd}$, i.e., according to the RHEED patterns the Cd-stabilized (111) $A$ surface remains smooth whereas the Cd-stabilized (111)B surface changes in about $10 \mathrm{~min}$. The evaporation rate of $\mathrm{Te}$ is much larger than that of $\mathrm{Cd}$, therefore, the evaporation of Cd should be the limiting factor as far as the surface structure is concerned.

\section{CONCLUSIONS}

In conclusion we have shown by means of XPS measurements and RHEED observations that CdTe (111) $A-(2 \times 2),(111) B-(1 \times 1)$ in a $C d$ environment, and (100)-c $(2 \times 2)$ are Cd-stabilized surfaces, and that CdTe (111) $A-(1 \times 1),(111) B-(1 \times 1)$ in a Te environment, and (100)- $(2 \times 1)$ are Te-stabilized surfaces. There is a mixed region between $\mathrm{Cd}$ and $\mathrm{Te}$ stabilization in which the RHEED patterns display both $\mathrm{Cd}$ - and Te-stabilized surface characteristics, and one is able to obtain a stoichiometric $\mathrm{CdTe}$ surface, i.e., the $\mathrm{Cd}$-to-Te ratio in the topmost monolayer is near 1 , if the proper fluxes and substrate temperature are employed.

The Cd-to-Te photoelectron-intensity ratio was used to determine the $\mathrm{Cd}$ fraction in the uppermost monatomic layer on the surface according to our simple model. Furthermore, the $\mathrm{Cd}$ surface coverage was correlated with the surface structure, i.e., the corresponding RHEED patterns. This correlation could in turn be employed to determine $\mathrm{Te}$ and $\mathrm{Cd}$ evaporation rates. The Te reevaporation rate is increasingly slower for the Testabilized (111) $A,(111) B$, and (100) surfaces, while the opposite it true for $\mathrm{Cd}$ from $\mathrm{Cd}$-stabilized (111) $\mathrm{A}$ and (111) $B$ surfaces.

The Cd-to-Te photoelectron-intensity ratio for the $3 d_{3 / 2}$ core levels, on (100) and (111) surfaces, is about $20 \%$ larger for a Cd-stabilized surface than for a Testabilized surface, independent of atomic sensitivity factors.

\section{ACKNOWLEDGMENTS}

This project was supported by the Bundesministerium für Forschung und Technologie and the Deutsche Forschungsgemeinschaft.
"On leave from Institute of Physics, Chinese Academy of Science, Beijing, China.

${ }^{1}$ G. L. Hansen, J. L. Schmitt, and T. N. Casselman, J. Appl. Phys. 53, 7099 (1982).

${ }^{2}$ S. Sivananthan, M. D. Lange, G. Monfroy, and J. P. Faurie, J. Vac. Sci. Technol. A 7, 788 (1988).

${ }^{3}$ R. D. Feldman M. Oran, R. F. Austin, and R. L. Opila, J. Appl. Phys. 63, 2872 (1988).

${ }^{4}$ T. H. Myers, R. W. Yanka, K. A. Harris, A. R. Reisinger, J. Han, S. Hwang, Z. Yang, N. C. Giles, J. W. Cook, Jr., J. F. Schetzina, R. W. Green, and S. McDevitt, J. Vac. Sci. Technol. A 7, 300 (1989).

${ }^{5}$ R J. Koestner, H.-Y. Liu, H. F. Schaake, and T. R. Hanlon, J. Vac. Sci. Technol. A 7, 517 (1989).

${ }^{6}$ J. P. Faurie, J. Reno, S. Sivananthan, I. K. Sou, X. Chu, M. Boukerche, and P. S. Wijewarnasuriya, J. Vac. Sci. Technol. B 6, 585 (1986).

${ }^{7}$ A. Sher, A.-B. Chen, W. E. Spicer, and C.-K. Shih, J. Vac. Sci. Technol. A 3, 105 (1985).
${ }^{8}$ J. H. Dinan and S. B. Quadri, J. Vac. Sci. Technol. A 3, 851 (1985).

${ }^{9}$ R. D. Feldman, R. F. Austin, A. H. Dayem, and E. H. Westerwick, Appl. Phys. Lett. 49, 797 (1986).

${ }^{10} \mathrm{G}$. Lentz, A. Ponchet, N. Magnea, and H. Mariette, Appl. Phys. Lett. 55, 2733 (1989).

${ }^{11}$ B. A. Joyce, Rep. Prog. Phys. 48, 1637 (1985).

12J. D. Benson, B. K. Wagner, A. Torabi, and C. J. Summers, Appl. Phys. Lett. 49, 1034 (1986).

${ }^{13}$ Y. S. Wu, A. Waag, and R. N. Bicknell-Tassius, Appl. Phys. Lett. 57, 1754 (1990).

${ }^{14}$ Y. S. Wu, C. R. Becker, A. Waag, R. N. Bicknell-Tassius, and G. Landwehr, J. Appl. Phys. 69, 268 (1991).

${ }^{15}$ S. Sivananthan, X. Chu, J. Reno, and J. P. Faurie, J. Appl. Phys. 60, 1359 (1986).

${ }^{16} \mathrm{C}$. Hsu, S. Sivananthan, X. Chu, and J. P. Faurie, Appl. Phys. Lett. 48, 908 (1986).

${ }^{17}$ A. Waag, Y. S. Wu, R. N. Bicknell-Tassius, and G. Landwehr, Appl. Phys. Lett. 54, 2662 (1989). 
${ }^{18}$ A. Waag, Y. S. Wu, R. N. Bicknell-Tassius, C. GonserBuntrock, and G. Landwehr, J. Appl. Phys. 68, 212 (1990).

${ }^{19}$ Y.-C. Lu, R. S. Feigelson, and R. K. Route, J. Appl. Phys. 67, 2583 (1990).

${ }^{20}$ M. Ekawa, K. Yasuda, S. Sone, Y. Sugiura, M. Saji, and A. Tanaka, J. Appl. Phys. 67, 6865 (1990).

${ }^{21}$ J. M. Arias, S. H. Shin, and E. R. Gertner, J. Cryst. Growth 86, 362 (1988).

22J. D. Benson and C. J. Summers, J. Cryst. Growth 86, 354 (1988).
${ }^{23}$ C. D. Wagner, W. M. Riggs, L. E. Davis, and J. F. Moulder, Handbook of X-ray Photoelectron Spectroscopy (Perkin-Elmer, Eden Prairie, MN, 1978); C. J. Powell, Surf. Sci. 44, 29 (1974).

${ }^{24}$ J.-P. Faurie, C. Hsu, and T. M. Duc, J. Vac. Sci. Technol. A 5, 3074 (1987); J. R. Waldrop, R. W. Grant, S. P. Kowalczyk, and E. A. Kraut, ibid. A 3, 835 (1985).

${ }^{25}$ C. B. Duke, A. Paton, W. K. Ford, A. Kahn, and G. Scott, Phys. Rev. B 24, 3310 (1981).

${ }^{26}$ A. Kahn, Surf. Sci. 168, 1 (1986). 